\title{
Reproducible dynamic dark ring lattices for ultracold atoms
}

\author{
N Houston, E Riis and A S Arnold \\ SUPA, Department of Physics, University of Strathclyde, Glasgow G4 0NG, UK \\ E-mail: a.arnold@phys.strath.ac.uk
}

Received 28 August 2008, in final form 11 September 2008

Published 13 October 2008

Online at stacks.iop.org/JPhysB/41/211001

\begin{abstract}
We demonstrate the generation of highly adaptable and reproducible dark optical ring lattices, which do not require Laguerre-Gauss beams or interferometric stability. In conjunction with a magnetic trap, these scanned 2D intensity distributions will enable low-decoherence trapping and straightforward dynamic manipulation of ultracold species in annular geometries using low-intensity regions of blue-detuned light. The technique is ideal for azimuthal ratchet, Mott insulator and persistent current experiments with quantum degenerate gases.
\end{abstract}

M This article features online multimedia enhancements

(Some figures in this article are in colour only in the electronic version)

\section{Introduction}

Laser cooling and the subsequent attainment of quantum degenerate gases has enabled exquisite control over atoms. Coherent atom-optical manipulation of coherent atomic gases, typically with magnetic fields and/or far-detuned lasers, is now a worldwide phenomenon [1]. There are a plethora of available atom trapping geometries and rings are of particular interest because they enable precision Sagnac interferometry and detailed studies of superfluidity, plus the periodic boundary conditions afford simple modelling of the system.

Bose-condensed atoms have recently been obtained in magnetic ring geometries with radii 1-50 $\mathrm{mm}$ [2] and persistent currents [3] have been observed in a small-scale condensate ring trap [4]. Ultracold atom ring traps based on electrostatic potentials [5] and induced currents [6] have also been proposed, and RF dressed rings [7] have been experimentally realized [8].

Confinement within optical lattice geometries offers further possibilities; potentially as a tool for performing universal simulation of quantum dynamics [9], and for realization of Feynman's ideas of quantum logic [10], thus forming a promising basis for quantum computation [11]. Optical lattices enable condensed matter physics investigations, such as the quantum phase transition from superfluidity to Mott insulator [12] and the realization of Josephson junction arrays [13]. The combination of rings and lattices to form ring lattices with rotational symmetry and periodic boundary conditions is particularly beneficial for e.g. studies of solitons, quantum many particle systems, entanglement, Mott transitions and persistent currents [14].

Ring lattice potentials utilize far-detuned optical dipole beams, which can be clearly divided into 'bright' or 'dark' lattices: atoms are attracted to dark (bright) spatial regions of the time-averaged optical potential if one uses light bluedetuned (red-detuned) from an atomic resonance. Bluedetuned light is clearly preferable for optical manipulation, as atoms trapped in low intensity light experience lower photon scattering (i.e. heating) rates, energy level shifts and light assisted collisional losses [15]. Blue-detuned ('dark') trapping ensures robustness against decoherence and will be essential for lattice-based quantum computation [11] and macroscopic superpositions of flow states in BEC ring lattices [16].

Dark optical ring lattices [17] have been previously realized using copropagating superpositions of LaguerreGauss (LG) laser beams [18]. Complex optics (spatial light modulators, SLMs) were used to generate separate static LG beams, which were then frequency shifted by independent acousto-optic modulators (AOMs). For a dynamic lattice the LG beams must be recombined on a beamsplitter, leading to 




Figure 1. Parametric curves described by equation (1) with $R=A+B \sin \omega_{2} t$ and $\left\{A / B, \omega_{2} / \omega_{1}\right\}$ set to: $\{1 / 2,5 / 2\}(\mathrm{a}) ;\{1,3 / 2\}$ (b); $\{1 / 2,1 / 6\}$ (c); $\{1 / 2,5\}$ (d). In (e) $R=A\left(1+0.3 \sin \left(6 \omega_{1} t\right)+\right.$ $\left.0.075 \sin \left(12 \omega_{1} t\right)\right)$ and the angular symmetry of the lattice is broken.

power loss and a requirement for interferometric relative beam stability to ensure reproducible long-term experiments. An alternative way to produce smooth time-dependent lattices is to update dynamically the SLM pattern; however update rates tend to be slow, and for the faster binary SLMs additional control algorithms must also be used, due to SLM interframe artefacts [19].

Bright ring lattices can be directly generated with AOMs. The acoustic mode frequency and amplitude in an AOM determine the beam deflection angle and intensity respectively, allowing spatial control [20]. By scanning the position of a laser beam in one or two dimensions it is possible to create a BEC mirror [21], condensate surface excitations and multiplesite traps [20], or 'stir' a condensate to produce vortices [22]. Two-dimensional bright ring lattice potentials using scanned red-detuned laser beams have been suggested [23], optically realized [24] and experimentally implemented for storing and splitting Bose-Einstein condensates [25].

Here we utilize two-dimensional AOM beam scanning $[20,24,25]$ to optically demonstrate simple dark optical ring lattice potentials for use with ultracold atoms or BECs, without the need for relative laser beam phase coherence required in [18], and with greatly reduced inferred atomic decoherence and heating compared to reference [24]. In BEC experiments using destructive imaging, the requirement of a ring lattice with a well-defined shot-to-shot angular phase is crucial and our potentials can be reproducibly rotated around the beam axis and also spatially modulated. Moreover, if additional confinement is provided by a magnetic field (section 4), a bluedetuned realization of the dark ring lattice will be essentially decoherence free [11] and highly adaptable-ideal for Mott insulator [12], persistent current [3] and azimuthal ratchet [26] experiments involving quantum degenerate gases. We will discuss the ring lattice theory, describe the experiment, then place our results in an atom optics context.

\section{Theory}

We consider ring-lattice potentials based on a laser beam following a $2 \mathrm{D}$ path of the form

$$
\left\{x_{c}, y_{c}\right\}=R\left\{\sin \left(\omega_{1} t\right), \cos \left(\omega_{1} t\right)\right\},
$$

where $R\left(\omega_{2} t+\phi\right)$ is an arbitrary function with period $2 \pi / \omega_{2}$ (i.e. $R$ is comprised of Fourier components at angular frequencies that are integer multiples of $\omega_{2}$ ). The overall period of the parametric function is $T=2 \pi / \omega_{\mathrm{gcd}}$ where $\omega_{\mathrm{gcd}}$ is the greatest common divisor of $\omega_{1}$ and $\omega_{2}$. Equation (1) forms a large family of rotationally symmetric 'flower like' curves (figure 1) defined by the Fourier components of $R$.
If $\omega_{2} / \omega_{1}$ is rational, $\omega_{2} / \omega_{\text {gcd }}$ corresponds to the number of 'petals' in the curve, which can be arbitrarily large in principle. Any curve can be directly rotationally modulated using the overall phase of $R$, i.e. $\phi$.

Half integer values of $\omega_{2} / \omega_{1}$ produce curves with a ring lattice profile (figure 1(a)), integer values of $\omega_{2} / \omega_{1}$ produce curves which, when combined with a magnetic quadrupole potential (section 4) create a hybrid magneto-optic ring lattice (figure 1(d)) and it is also possible to make patterns with broken angular symmetry for ring ratchet experiments (figure 1(e)).

Laser beam deflection from an AOM is proportional to the RF drive frequency. The deflection can be modulated by altering the drive RF frequency, which can be achieved by varying the voltage driving a voltage controlled oscillator (VCO). Thus AOM deflection is synchronized to the VCO input voltage. If a laser beam is passed through two perpendicular AOMs, with corresponding VCOs driven by the parametric signal equation (1), the variation in the vertical and horizontal deflection angles will cause the beam to trace out the corresponding parametric curves. If this variation is rapid compared to typical atomic velocities, ultracold species will effectively experience only the time-averaged geometry of the beam trace [27], allowing both trapping and spatial control of atoms or BECs within the scanned beam.

\section{Experiment}

The design used to generate the required VCO control signals is shown schematically in figure 2 . The circuit is able to operate from a single 'master' synthesized signal generator $\left(\mathrm{SSG}_{\mathrm{M}}\right.$, e.g. Agilent $33220 \mathrm{~A}$ or SRS DS345) by taking advantage of the $10 \mathrm{MHz}$ clock output, to synchronize a second 'slave' $\mathrm{SSG}_{\mathrm{S}}$ (or control circuitry [28]). $\mathrm{SSG}_{\mathrm{M}}$ is used for radial modulation of the circular beam path provided by $\mathrm{SSG}_{\mathrm{S}}$. We have used analogue multipliers because the amplitude modulation function of a typical SSG has limited bandwidth.

The multiplier outputs (equation (1)) are then fed to two independent VCOs to provide the RF signals for driving two $110 \mathrm{MHz}$ AOMs. Voltage-controlled attenuators (VCAs) or double-balanced mixers are then used for control over RF power, and thus beam intensity, during the beam scan. Our base frequency is currently set to $\omega_{1}=2 \pi \times 10 \mathrm{kHz}$ (a subharmonic of the $\mathrm{SSG}_{\mathrm{M}}$ clock) for convenience, however with sufficient VCO bandwidth, this could easily be increased by more than a factor of 10 .

Figure 3 shows the experimental setup. A helium-neon laser beam is focused through two perpendicular AOMs, which are placed close to each other to minimize output distortion and scan asymmetry. Each AOM produces a spread of diffractive orders which results in a grid of diffracted beams at the output.

Figure 4 shows experimental results for 5-site optical ring lattices realized using this technique, as well as comparison to 2D least-squares fits from a simple theoretical model based on a Gaussian beam with $x$ and $y$ beam waists of $w_{x}$ and $w_{y}$ respectively, scanned in the $x y$-plane yielding a time-averaged 


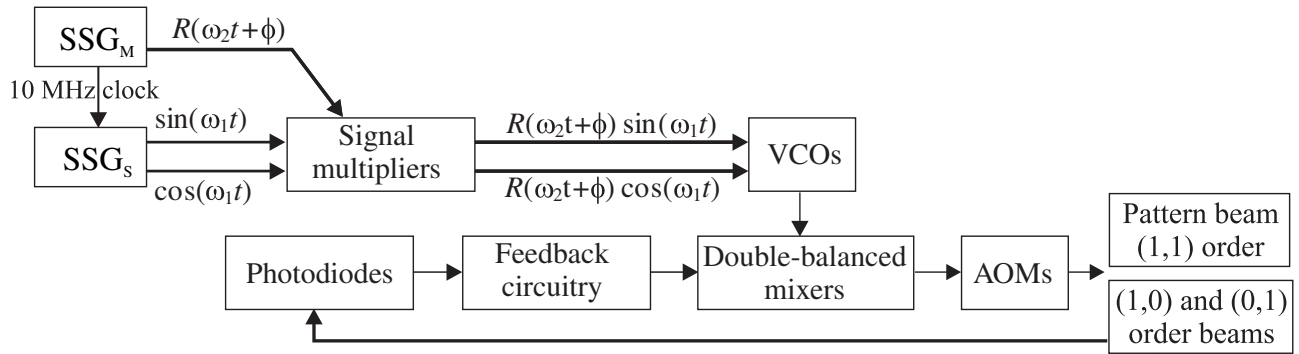

Figure 2. The signal generation circuit is based on two synthesized signal generators (SSG). Our slave synthesizer $\mathrm{SSG}_{\mathrm{S}}$ is actually a custom-made circuit based on low-cost ICs [28].

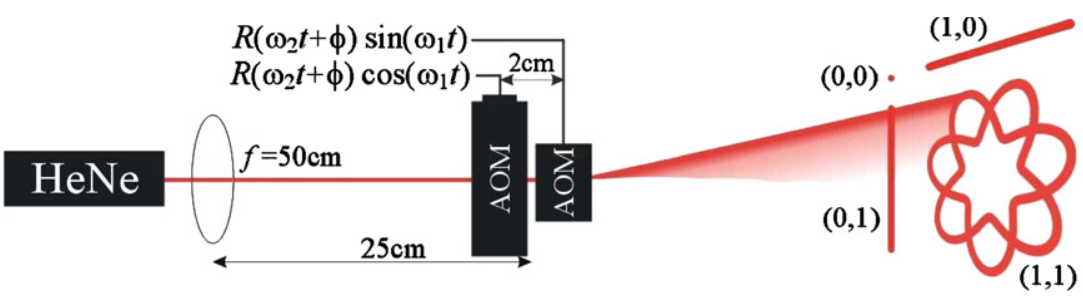

Figure 3. Experimental setup for generation of optical ring lattices. Synchronized variation in AOM deflection angles causes the beam to trace out the ring lattice shown.
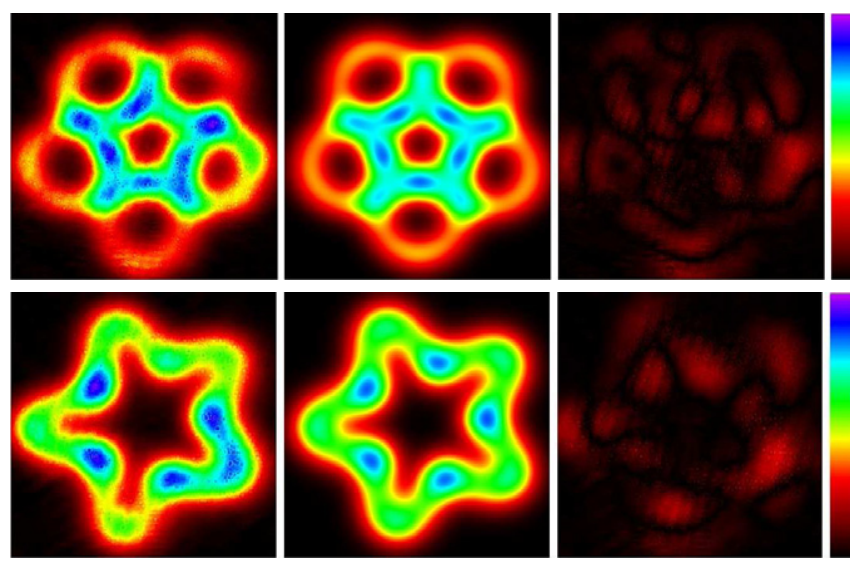

Figure 4. Experimental relative intensity distributions (left; area $\approx(4 \mathrm{~mm})^{2}$, exposure $\left.1 \mathrm{~ms}\right)$, corresponding least-squares theoretical fits using equation (2) (centre) and fit residues (right). Optical lattice movies comparing experimental and theoretical rotation (i.e. $\phi$ ) and amplitude (i.e. $A_{x, y}$ ) modulation are available at

stacks.iop.org/JPhysB/41/211001/mmedia. The static laser beam has a waist $\left(e^{-2}\right.$ radius $) \approx 300 \mu \mathrm{m}$.

intensity:

$\overline{I(x, y)}=\frac{I_{0} \omega_{\mathrm{gcd}}}{2 \pi} \int_{0}^{\frac{2 \pi}{\omega_{\mathrm{gcd}}}} \exp \left[\frac{\left(x-x_{c}\right)^{2}}{w_{x}^{2}}+\frac{\left(y-y_{c}\right)^{2}}{w_{y}^{2}}\right]^{-2} \mathrm{~d} t$,

where $x_{c}=x_{0}-\left(r_{x}+A_{x} \sin \left(\omega_{2} t+\phi\right)\right) \sin \left(\omega_{1} t\right)$ and $y_{c}=$ $y_{0}-\left(r_{y}+A_{y} \sin \left(\omega_{2} t+\phi\right)\right) \cos \left(\omega_{1} t\right)$ are the $x$ and $y$ position of the centre of the scanned beam at time $t$, respectively (cf equation (1)). Note that $\omega_{\mathrm{gcd}}=\omega_{1}$ for integer $\omega_{2} / \omega_{1}$ (an open optical ring lattice cf figure 1(a)) whilst $\omega_{\text {gcd }}=$ $\omega_{1} / 2$ for half-integer $\omega_{2} / \omega_{1}$ (a closed optical ring lattice cf figure $1(\mathrm{~d})$ ) respectively. The ten variables used in the leastsquares fits were $x_{0}, y_{0}, w_{x}, w_{y}, r_{x}, r_{y}, A_{x}, A_{y}, I_{0}$ and $\phi$.
As the contribution to the time-averaged intensity is inversely proportional to the velocity of the centre of the scanned beam, there is a radial intensity gradient due to the deflected beam spending longer in the centre of the pattern. This gradient does not affect the quality of the trapping potential for e.g. Mott insulator experiments, as long as the pattern has a high level of rotational symmetry. It is therefore important to scan a dipole beam with an aspect ratio as close as possible to 1 , with a stable beam intensity during the scan process. To this end, we have implemented a feedback mechanism ('noise-eater') to the VCA control signals based on error signals generated from the unused $(1,0)$ and $(0,1)$ AOM deflection orders (figure 3 ). The advantage of feedback, as opposed to recorded feedforward [24], is that it immediately adapts to beam intensity noise due to environmental changes or changes to the amplitude modulation function. Although high bandwidth is required for feedback, we have already reduced intensity amplitude noise to $5 \% \mathrm{rms}$, and anticipate future improvements.

\section{Atom optics}

Whilst there has been growing theoretical interest in ring lattices [14], the closest experiment to a dark BEC ring lattice has consisted of three sites produced by variable intensity light traversing a static mechanical aperture [29]. The azimuthal and central barriers of this 3-site ring lattice must be altered together. In contrast the azimuthal and central barrier of our $N$-site optical potential can be altered independently. Additionally, as the azimuthal lattice angle is purely controlled by the phase $\phi$ of the VCO amplitude modulation, the ring lattice can be reproducibly rotated, even for BECs with experimental production times of minutes.

We now give a brief illustration of experimentally realistic parameters for a 5-site optical ring lattice. The scanned laser 

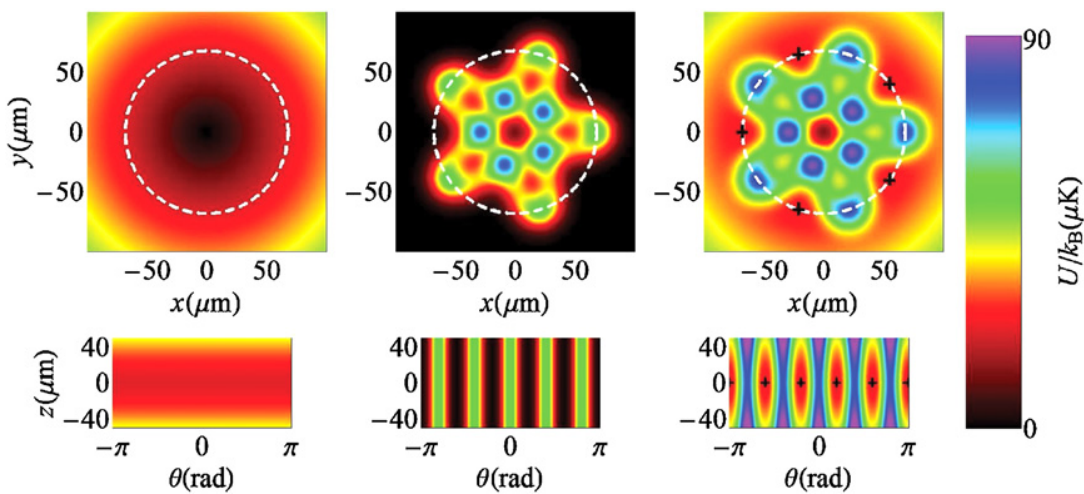

Figure 5. Magnetic (left), optical (center) and hybrid magneto-optical potentials (right). Upper images show the $z=0$ radial plane and the lower images show axial-azimuthal slices indicated by the dashed white circles. Lattice sites in 3D (black crosses) are only formed for the magneto-optical potentials.

beam leads to an optical dipole trap with atomic scattering rate $(\mathrm{Hz})$ and depth $(\mathrm{J})$ approximately given by $S \approx \Gamma \bar{I} /\left(8 I_{\mathrm{S}} \Delta_{\Gamma}{ }^{2}\right)$ and $U \approx \hbar \Gamma \bar{I} /\left(8 \Delta_{\Gamma} I_{\mathrm{S}}\right)$ respectively, where $\bar{I}$ and $\Delta_{\Gamma}=$ $\left(\omega-\omega_{0}\right) / \Gamma$ are the time-averaged spatial intensity and laser detuning from the atomic transition (in linewidths). A very useful, species independent parameter for blue-detuned dipole traps is $S / T=k_{\mathrm{B}} /\left(\hbar \Delta_{\Gamma}\right)$ - the maximum scattering rate experienced by an atom with total energy $U=k_{\mathrm{B}} T$ as a function of the dipole trap depth. For the $780 \mathrm{~nm} \mathrm{D} 2$ transition in ${ }^{87} \mathrm{Rb}, \Gamma=2 \pi \times 6 \mathrm{MHz}$ and $I_{\mathrm{S}}=16.7 \mathrm{~W} \mathrm{~m}^{-2}$. If we use a dipole trap laser with wavelength $\lambda=765 \mathrm{~nm}$ $\left(\Delta_{\Gamma} \approx 1.3 \times 10^{6}\right)$ we have $S / T=0.1 \mathrm{~Hz} \mu \mathrm{K}^{-1}$. Assuming a laser waist of $w=20 \mu \mathrm{m}$, and power $P=400 \mathrm{~mW}$, figure 5 illustrates the optical dipole potential, as well as its combination with a magnetic quadrupole field $\mathbf{B}=$ $100 \mathrm{G} \mathrm{cm}^{-1}\{-x / 2,-y / 2, z\}$ for atoms in the ground state $\left|F=2, m_{F}=2\right\rangle$, yielding an adiabatic magnetic potential $U_{B}=\mu_{B}|\mathbf{B}|[1]$.

In addition to the dramatically reduced heating rates afforded by blue-detuned (dark) dipole traps, it should be stressed [20] that dark ring lattices should greatly reduce the observed heating due to micromotion of atoms during AOM beam scan in bright dipole traps. Additionally, scattering a few photons tends to optically pump atoms into magnetically untrapped states: thus dark (bright) traps scatter more photons in the hottest (coldest) parts of the potential leading to evaporative cooling (heating).

\section{Conclusions}

We have experimentally obtained reproducible optical ring lattices for use with blue-detuned light, which allow controllable rotation and spatial modulation. The lattices produced are relatively insensitive to environmental conditions and do not require either LG beams, SLMs or other complex optics. This technique could possibly be utilized for rotation of a 'quantum register', and for new forms of optical tweezing.

\section{Acknowledgments}

We gratefully acknowledge discussions with Dr Malcolm Boshier and Dr Ifan Hughes.

\section{References}

[1] Hinds E A and Hughes I G 1999 J. Phys. D: Appl. Phys. 32 R119

Adams C S and Riis E 1997 Prog. Quantum Electron. 211 (Ultracold atom network, http://ucan.physics.utoronto.ca)

[2] Gupta S, Murch K W, Moore K L, Purdy T P and Stamper-Kurn D M 2005 Phys. Rev. Lett. 95143201

Arnold A S, Garvie C S and Riis E 2006 Phys. Rev. A 73041606

[3] Ryu C et al 2007 Phys. Rev. Lett. 99260401

[4] Naik D S, Muniz S R and Raman C 2005 Phys. Rev. A 72051606

[5] Hopkins A, Lev B and Mabuchi H 2004 Phys. Rev. A 70053616

[6] Griffin P F, Riis E and Arnold A S 2008 Phys. Rev. A 77051402

[7] Morizot O et al 2006 Phys. Rev. A 74023617

Fernholz T et al 2007 Phys. Rev. A 75063406

Lesanovsky I and von Klitzing W 2007 Phys. Rev. Lett. 99083001

[8] Hofferberth S et al 2006 Nature Phys. 2710

Heathcote W H et al 2008 New J. Phys. 10043012

[9] Jané W, Vidal G, Dür W, Zoller P and Cirac J I 2003 Quantum Inform. Comp. $\mathbf{3} 15$

[10] Feynman R P 1982 Int. J. Theor. Phys. 21467

[11] Kay A, Pachos J K and Adams C S 2006 Phys. Rev. A 73022310

[12] Greiner M, Mandel O, Esslinger T, Hänsch T W and Bloch I 2002 Nature 41539

[13] Cataliotti F S et al 2001 Science 293843

[14] Plaja L and San Román J 2004 Phys. Rev. A 69063612

Amico L, Osterloh A and Cataliotti F 2005 Phys. Rev. Lett. 95063201

Rey A M et al 2007 Phys. Rev. A 75063616

Wang T, Javanainen J and Yelin S F 2007 Phys. Rev. A 76011601

[15] Ozeri R, Khaykovich L and Davidson N 1999 Phys. Rev. A 59 R 1750

[16] Hallwood D and Dunningham J 2006 Phys. Rev. A 74023601 Hallwood D W, Burnett K and Dunningham J 2006 New J. Phys. 8180

[17] Chattrapiban N, Rogers E A, Arakelyan I V, Roy R and Hill W T 2006 J. Opt. Soc. Am. B 2394

[18] Franke-Arnold S et al 2007 Opt. Express 158619

[19] Boyer V, Chandrashekar C M, Foot C J and Laczik Z J 2004 J. Mod. Opt. $\mathbf{5 1} 2235$

Boyer V et al 2006 Phys. Rev. A 73031402

[20] Onofrio R et al 2000 Phys. Rev. Lett. 84810 
[21] Bongs K et al 1999 Phys. Rev. Lett. 833577

[22] Madison K W, Chevy F, Wohlleben W and Dalibard J 2000 Phys. Rev. Lett. 84806

[23] Schwartz S, Cozzini M, Menotti C, Carusotto I, Bouyer P and Stringari S 2006 New J. Phys. 8162

[24] Schnelle S K, van Ooijen E D, Davis M J, Heckenberg N R and Rubinsztein-Dunlop H 2008 Opt. Express 161405

[25] Boshier M G 2007 private communication
[26] Gommers R, Lebedev V, Brown M and Renzoni F 2008 Phys. Rev. Lett. 100 040603, and references therein

[27] Petrich W, Anderson M H, Ensher J R and Cornell E A 1995 Phys. Rev. Lett. 743352

Arnold A S 2004 J. Phys. B: At. Mol. Opt. Phys. 37 L29

[28] Houston N, Riis E and Arnold A S (in preparation)

[29] Scherer D R, Weiler C N, Neely T W and Anderson B P 2007 Phys. Rev. Lett. 98110402 